\title{
Ideen, Institutionen, Interessen
}

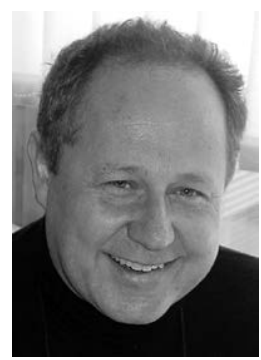

Manfred Wildner

\section{Korrespondenzadresse}

Prof. Dr. med. Manfred Wildner

Bayerisches Landesamt für Gesundheit und Lebensmittel-

sicherheit

Veterinärstraße 2

85764 Oberschleißheim

Manfred.Wildner@lgl.bayern.de

Bibliografie

DOI https://doi.org/10.1055/a-1001-2853

Gesundheitswesen 2019; 81: 862-863

(c) Georg Thieme Verlag KG Stuttgart · New York

ISSN 0941-3790
Platon, der große Philosoph und erste „Akademiker“, hatte beste Absichten, als er das Höhlengleichnis entwarf. Er stellte damit das Leben von Gefangenen in der „Höhle“ des Materiell-Diesseitigen, in welcher man lediglich über Schatten von den eigentlichen Vorgängen im Licht außerhalb erfährt, einem befreiten Leben in diesem Licht der geistigen Erkenntnis gegenüber: ein durch Philosophie entfaltetes Leben im Land der Ideen, des Guten, Wahren und Schönen [1]. Raffael stellte 2000 Jahre später Platon in die Mitte seines Freskos „Die Schule von Athen“, gemalt an eine Decke im Vatikan. Allerdings steht dort neben den mit dem Zeigefinger zum (Ideen-) Himmel deutenden Platon sein Schüler Aristoteles, dessen Hand auf den Boden zeigt: in das Hier und Jetzt, auf die Welt der Dinge und Fakten und das in ihr ethisch Gebotene.

So können wir, mit ästhetischem Genuss und auf hohem Niveau von diesem Paradox verwirrt, nun darüber nachsinnen, was denn gilt: die reinen Ideen oder die „störrischen Fakten“ (stubborn things), um mit John Adams, dem zweiten Präsidenten der USA zu sprechen (s.a. [2]). Jedenfalls sind es zumindest im nachchristlichen akademischen Konsens nicht die „alternativen Fakten“ (vulgo: blanke Lügen) der Sprechweise von Kellyanne Conway, der Beraterin des 45. amerikanischen Präsidenten, die gelten sollten. Aber ist die Wahrheit denn so einfach zu finden? Sind sogenannte Fakten nicht immer auch Konstrukte in den Augen bzw. dem zentralen Nervensystem des Betrachters/der Betrachterin, die oft genug korrigiert werden müssen? Der Philosoph Geert Keil weist scharfsichtig darauf hin, dass es unsere Aussagen über die Tatsachen sind, die wahr, falsch oder vorläufig sein können, nicht die Tatsachen selbst. Tatsachen sind, was sie sind, auch wenn sie nicht buchstäblich fassbare Dinge sind, sondern „abstrakte Gegenstände“. Sie sind damit hinsichtlich unserer immer potentiell fehlbaren wissenschaftlichen und sozialen Konstruktionen oft „störrische Dinge“ [2].

Ist angesichts dieser potentiellen Fehlbarkeit wissenschaftlichen Aussagen überhaupt noch zu trauen? Leben wir, angesichts dieser Vorläufigkeit, nicht unvermeidlich in einer von einer postfaktischen Beliebigkeit geprägten Welt und damit in einer großen wissen- schaftlichen Vertrauenskrise? Diese Weltsicht mag den „harten“ Naturwissenschaften, allen voran der Physik, fremd sein, deren Erkenntnis ganze Städte zuverlässig mit Energie zu versorgen vermag. Dem damit vielleicht indirekt gescholtenen Bereich der „weichen" Geistes- und Sozialwissenschaften liegt eine solche Aussage allerdings nicht so fern. Mit Süffisanz weist z. B. das Thomas-Theorem darauf hin, dass unsere Vorstellungen durchaus irreal sein können, die Konsequenzen aus unbegründeten Hoffnungen und Ängsten jedoch sehr wohl real sind bzw. werden [3] - nachprüfbar z. B. an Börsenkursen und Wahlergebnissen als sozial konstruierten Realitäten.

Die Soziologen André Kieserling und Simone Rödder regen zu dieser Vertrauenskrise in einem vermeintlich „postfaktischen“ Zeitalter eine unaufgeregte und balancierte Bewertung an und verweisen auf eine dreifache Schwäche dieser These [4]. Zum einen ist es die, für Zeitdiagnosen wohl typische, falsche Beschreibung der Vergangenheit, welche die überhaupt nicht neue Verweigerung gegenüber Tatsachen z. B. in Ideologien und religiösem Fundamentalismus übersieht. Zum zweiten ist es der Irrtum, dass v. a. über die Tatsachen gestritten würde: Gegenstand der Meinungsverschiedenheiten sind meist die Deutungen von Tatsachen und die sich darauf stützenden normativen bzw. politisch-weltanschaulichen Aussagen. Zum dritten ist hier ein Trugschluss wirksam, der das Vertrauen in die Wissenschaft zu sehr nach dem Muster des zwischenmenschlichen Vertrauens denkt. Gegenüber wissenschaftlichen Erkenntnis(aussagen) ist es durchaus möglich, in einzelnen Fällen misstrauisch zu sein, ohne deswegen das grundsätzliche Vertrauen in die Wissenschaft in Frage zu stellen. Diese bleibt die breit akzeptierte Grundlage für weite Teile unseres praktischen Lebens, beispielsweise in der Telekommunikation, im Bereich der Mobilität oder in der modernen Medizin.

Diese praktische Weltgestaltung gründet allerdings in einer Welt der Ideen, welcher naturwissenschaftliche Tatsachenaussagen bzw. Theorien genauso entspringen wie gesellschaftliche Ideen über Demokratie, Freiheit, soziale Gerechtigkeit. Auf Basis von Ideen können so im weiteren Institutionen entstehen, welche Prozesse 
in Gang setzen, erhalten und moderieren, wie die Institutionen einer Universität, einer staatlichen Netzagentur oder eines Krankenhauses. Ideen und Institutionen bringen wiederum auch Akteursinteressen und Fragen nach Macht und Einfluss hervor, welche in Beziehungsnetzen wirksam und ausgehandelt werden. Auf dieses Konzept der drei „।“ (Ideen, Institutionen, Interessen) wird bspw. in Politik- und Stakeholderanalysen zurückgegriffen [5, 6].

Wenn dies auch für das Gesundheitswesen als komplexes gesellschaftliches Netzwerk gilt: was wären hier die Akteure („Stakeholder“) bzw. die wesentlichen Institutionen, ihre zu Grunde liegenden Leitideen und ihre konkreten Interessen? In einem früheren Beitrag wurde schon auf das Akteursdreieck aus Patienten bzw. Versicherten („patients/people“), direkten und indirekten Leistungserbringern („,provider“) und Financiers („,payers“, also Versicherungen, private Investoren, staatliche Organe) hingewiesen, ergänzt um die Ebene der politischen und wissenschaftlich-fachlichen Steuerung zu einer Akteurspyramide [7]. Diese Heuristik ist jedoch nur ein erster Schritt. Um als Beispiel die Diskussion um eine (Masern-) Impfpflicht aufzugreifen: Legitime tragende Ideen wären z. B. bei den Institutionen der Leistungserbringer das Wohl der Patienten und die wissenschaftliche Evidenz, mögliche konkrete Interessen könnten Deutungshoheiten, ökonomische Gewinne oder die Compliance mit politischen Vorgaben wie die einer weltweiten Masernausrottung sein. Auf Patienten- bzw. Versichertenseite könnte die Idee von Freiheit - Freiheit auch von Krankheit - tragend sein, das (Kinder-)Recht auf ein Leben in Würde und in Selbstbestimmung. Konkrete Interessen könnten die Kostenfreiheit einer solchen Impfung sein, der Schadensersatz bei Impfkomplikationen und nicht zuletzt auch die Entscheidungsfreiheit gegenüber einer Impfung. Auf Seiten der Financiers sind die Leitideen u. a. in den Sozialgesetzbüchern formuliert, allen voran Humanität, Qualität und Wirtschaftlichkeit (§70 SGB V), aus denen auch konkrete Interessen ableitbar sind. Politisch sind nun diese teilweise konvergenten, teilweise kontroversen Leitideen und konkretisierten Interessen zu verhandeln, in den Kontext einer freiheitlichen demokratischen Grundordnung zu setzen und zu einem tragfähigen Ausgleich zu bringen. Das jedenfalls wären typische Interessen einer von den Ideen von Demokratie und Rechtsstaatlichkeit geprägten gesellschaftlichen Ordnung. Klingt kompliziert? Es kommt noch schlimmer: Nicht immer laufen die Dinge so ideal, dem (Ideen-)Himmel sei's geklagt.

Die Kompliziertheit ist dabei auch der Komplexität der zu Grunde liegenden, vielfältig interagierenden „abstrakten Gegenstände“ einer modernen Gesellschaft geschuldet. Eines Gemeinwesens, das gerade deswegen gut beraten ist, sich seiner gesellschaftlichen Grundwerte wie Wahrheit, Gleichheit und weitergehenden Werten sozialer Gerechtigkeit immer wieder zu versichern und sie auch ggf. einzufordern. „Der freiheitliche, säkularisierte Staat lebt von Voraussetzungen, die er selbst nicht garantieren kann. Das ist das große Wagnis, das er, um der Freiheit willen, eingegangen ist", lautet das Diktum des Verfassungsrichters Ernst-Wolfgang Böckenförde [8]. Und so ist in einer aufgeklärten und freien Gesellschaft und seinem Gesundheitswesen immer wieder neu die Stärke des Rechts zu suchen, nicht das Recht des Stärkeren und dem zwanglosen Zwang des besseren Arguments der Vorzug zu geben vor dem verführerisch kurzen Weg der Interessensdurchsetzung mit (staatlicher) Gewalt. Herrschaftliche Gewalt muss um der Freiheit willen immer wieder weise geteilt und klug eingehegt werden.
Auch diese Ausgabe will wieder Beiträge zu den wissenschaftlichen und sozialen Grundlagen eines in Vielfalt einigen und zukunftsoffenen Gemein- und Gesundheitswesens liefern: Mit Arbeiten zur Bürgerorientierung bei der Themenauswahl für HTA-Berichte, zu Verhaltensauffälligkeiten im Vorschulalter in Hannover, zu körperlicher Aktivität im Kontext der betrieblichen Gesundheitsförderung, zu Hintergründe der Langzeiteinnahme von Benzodiazepinen und Z-Substanzen bei älteren Patienten, zu Einflussfaktoren auf die distanzbezogene Arztwahl am Beispiel von Patienten mit Psoriasis und chronischen Wunden, zur Inanspruchnahme ambulanter Leistungen bei Arthrose, rheumatoider Arthritis oder Osteoporose, zu einem Fragebogen zur Erfassung der musikalischen Aktivität, zur Evaluierung der mundgesundheitsbezogenen Lebensqualität bei seltenen Erkrankungen, zum Informationsverhalten der Südtiroler Bevölkerung zu Gesundheitsfragen, zum kalkulatorischen Arztlohn im EBM, zum Lübecker Hochschulmodell der Physiotherapie sowie mit einer Richtung gebenden sozialmedizinischen Grundlagenarbeit in 2 Teilen zum Begriff der medizinischen Notwendigkeit.

Um noch einmal zu Raffaels Paradox zurückzukommen: Wie verhält es sich nun um das Spannungsfeld von Ideen und Fakten, welchem der beiden Felder ist der Vorzug zu geben? Die Naturwissenschaften deuten auf den Boden der Tatsachen: auf das Primat des Ontischen, Seienden, vor jeder geistiger Ideen-schaffenden Tätigkeit. Erst das Gehirn, dann der Gedanke. Und sie stoßen doch gleichzeitig an die Grenzen des Materiellen: Einer Materie, deren Elementarteilchen sich in mathematisch-ideale Formeln, nicht unähnlich geistigen Ideen, aufzulösen scheinen und welche sich auch in der anderen Richtung zunehmender Komplexität, z. B. bei komplexen biologischen und sozialen Organisationen von einem materiellen Determinismus löst und zu Ideen, Interessen, Institutionen und ihren vielfältigen Interaktionen führt. Die Antwort auf Raffaels Paradox findet sich vermutlich auch gar nicht in der Frage nach einem „Entweder-oder“, sondern vielmehr in der Einsicht in ein notwendiges „Sowohl-als-auch“. Einer Achtsamkeit in beiden Bereichen: weil aus Ideen und Interessen Tat(sach)en werden können und daraus individuelle und gesellschaftliche Schicksale, unser eigenes inbegriffen.

\section{Literatur}

[1] Platon. Politeia. Der Staat. Siebentes Buch. URL http://www. opera-platonis.de/Politeia7.pdf. download 16.10.2019

[2] Keil G. Über Tatsachen. Forschung \& Lehre 2019; 10: 895-897

[3] Thomas WI. The Methodology of Behavior Study. Chapter 13 in: The Child in America: Behavior Problems and Programs.Alfred A. Knopf; New York: 1928: 553-576

[4] Kieserling A, Rödder S. Misstrauen ohne Folgen. Forschung \& Lehre 2019; 10: 898-899

[5] Shearer JC, Abelson J, Kouyate B et al. Why do policies change? Institutions, interests, ideas and networks in three cases of policy reform. Health Policy and Planning 2016; 31: 1200-1211

[6] Walt G. Health Policy: An Introduction to process and power. Witwatersrand University Press; Johannesburg: 1994

[7] Wildner M. Vorhang auf! Gesundheitswesen 2019; 81: 525-526

[8] Böckenförde EW. Die Entstehung des Staates als Vorgang der Säkularisation. In: Recht, Staat, Freiheit. Suhrkamp; Frankfurt a.M: 1991: 112 